\section{Análise dos custos do procedimento PET-TC com 18F-FDG na perspectiva do SUS provedor: estudo em uma unidade pública de saúde do Rio de Janeiro, Brasil}

\author{
Cost analysis of 18 F-FDG PET-CT from the \\ perspective of the Brazilian Unified National Health \\ System as healthcare provider: a study in a public \\ healthcare center in Rio de Janeiro, Brazil
}

\author{
Análisis de costes del procedimiento PET-TC con \\ 18F-FDG desde la perspectiva del SUS proveedor: \\ estudio en una unidad pública de salud \\ de Río de Janeiro, Brasil
}

\begin{abstract}
Positron emission tomography (PET) has been introduced recently in Brazil and requires costs analysis to support economic evaluation studies on its use. The current study analyzed the use of 18 F-FDG PET-CT and estimated its costs from the perspective of a public healthcare provider. The micro-costing technique was used, identifying, quantifying, and valuing all the inputs used to perform the procedure. Cost estimates considered 85 tests performed at the Brazilian National Cancer Institute from March to June 2012. Reference cases were defined as adult cancer patients, output of five tests per day, and one dose of $18 \mathrm{~F}$ FDG per patient. Unit cost for the procedure was $B R L$ 3,150.30 based on career wages under the Ministry of Science and Technology and BRL 2,927.19 based on Ministry of Health career wages. The factor with the heaviest cost impact was daily output of tests. Other factors that could impact the test's cost in public healthcare institutions were also examined.
\end{abstract}

Positron-Emission Tomography; Biomedical Technology; Costs and Cost Analysis; High-Cost Technology
Rosângela Caetano 1

Luciene Fontes Schluckebier 2

Cláudia Regina Garcia Bastos 1

Rondineli Mendes da Silva 1

Michel Pontes Carneiro 2

Jorge Wagner Esteves da Silva 2

Aline Navega Biz ${ }^{1}$

\section{Resumo}

A tomografia de emissão de pósitrons (PET) é de uso recente no Brasil e há necessidade de se estimar os custos do procedimento, de forma a subsidiar estudos de avaliação econômica sobre a tecnologia. O trabalho analisou o processo de produção da PET-TC utilizando 18 F-FDG e estimou seus custos na perspectiva de um provedor público de serviços de saúde. Utilizouse a técnica de microcustos, com identificação, quantificação e valoração de todos os insumos consumidos na produção do procedimento. As estimativas de custo consideraram a observação de 85 exames realizados no Instituto Nacional de Câncer, de março/julho de 2012. O caso de referência considerou pacientes oncológicos adultos, volume de produção de 5 exames/dia e uso de uma dose de ${ }^{18} \mathrm{~F}-\mathrm{FDG}$ por paciente. Os custos unitários do procedimento foram de $R \$ 3.150,30$, na perspectiva salarial da carreira de Ciência \& Tecnologia, e de R\$2.927,19 na do Ministério da Saúde. O elemento de maior impacto nos custos correspondeu ao volume diário de produção dos exames. Foram explorados elementos que podem impactar no custo do exame nas instituições públicas de saúde.

Tomografia de Emissão de Pósitrons; Tecnologia Biomédica; Custos e Análise de Custo;

Tecnologia de Alto Custo 


\section{Introdução}

Em um mundo onde a contínua introdução de novas tecnologias tem sido acompanhada por um aumento constante nos gastos globais no setor saúde, análises econômicas são ferramentas importantes para o uso racional e eficiente dos recursos 1. Sua relevância é ratificada na Política Nacional de Gestão de Tecnologia em Saúde 2 e na Lei no $12.401^{3}$, que reforçam que as tomadas de decisão relativas às tecnologias em saúde devem basear-se em evidências científicas sobre eficácia, acurácia, segurança, e na avaliação econômica comparativa dos benefícios e custos em relação às já incorporadas.

A tomografia de emissão de pósitrons ( $p o$ sitron-emission tomography - PET) é uma tecnologia da área de medicina nuclear, complexa, de alto custo, que tem sido proposta, de forma adicional às técnicas de imagem anatômica, em diversas aplicações oncológicas. De difusão recente, com poucos equipamentos instalados na rede pública de saúde, estes procedimentos não fazem parte das tabelas de reembolso do Sistema Único de Saúde (SUS). Avaliações econômicas dessa tecnologia vêm sendo demandadas pelo Ministério da Saúde, com vistas a orientar suas decisões relacionadas à incorporação do procedimento ao sistema público de saúde.

Para a realização de estudos de custo-efetividade de base local e na perspectiva do SUS, estimativas dos custos das intervenções são essenciais. Estudos de custos do procedimento PET são escassos na literatura internacional 4,5,6 e inexistem em nosso meio, sendo importante estimativas baseadas em dados nacionais, que auxiliem no estabelecimento de valores de referência.

Os custos da tecnologia representam o valor de todos os recursos utilizados na sua produção e distribuição, como tempo, espaço físico, recursos humanos, bens de capital e materiais de consumo ${ }^{7}$. Estes valores variam de acordo com a perspectiva escolhida para análise, qual seja, a do provedor do serviço, financiador da assistência, pacientes e famílias etc.

Métodos de estimativa de custos baseados em atividade vêm sendo amplamente utilizados nas estimativas de custeio de serviços públicos, em particular em áreas como serviços laboratoriais e de diagnóstico por imagens ou de cuidados intensivos ${ }^{8}$. Seu princípio básico contempla uma análise orientada pelas atividades do processo de produção geradoras dos custos ${ }^{9}$, com realização de inventário detalhado dos recursos consumidos, sua quantificação e valoração 10 .

O estudo estimou os custos unitários do procedimento com PET-TC (tomografia computa- dorizada) na perspectiva de um provedor público de serviços, objetivando subsidiar os processos de decisão relativos à incorporação da tecnologia no rol de procedimentos oferecidos pelo SUS.

\section{Metodologia \\ Método de custeio}

Foi adotado para as estimativas o método de microcustos, que consiste em um inventário e mensuração detalhados de todos os recursos consumidos na provisão de uma intervenção de saúde. Este método é considerado a primeira opção em situações onde a intervenção é nova e não houve oportunidades de calcular um custo médio 11.

Dentre as diversas técnicas que podem ser empregadas nesse método, foi utilizado o custeio baseado em atividade (activity-based costing - ABC), que implicou a identificação das etapas de execução do procedimento PET, a quantificação dos recursos consumidos em cada uma delas e sua valoração 12 .

\section{Perspectiva e lócus do estudo}

Foi adotada a perspectiva do SUS como provedor de serviços. Os custos foram estimados com base em procedimentos realizados no Hospital do Câncer I, unidade do Instituto Nacional de Câncer (INCA). Esta unidade possui um equipamento de PET-TC dedicado (modelo GEMINI TF w/ToF, Philips Medical Systems, Eindhoven, Holanda), em operação desde setembro de 2009. Seu elevado desempenho possibilita o uso de atividades mais baixas do radiofármaco e menor exposição à radiação de pacientes e equipe. O curto tempo de aquisição e alta qualidade de imagem permitem que o serviço trabalhe com uma prática de aproveitamento de dose, possibilitando a produção diária com consumo menor de 18F-FDG (radiofármaco $18 \mathrm{~F}$-fluorodeoxiglicose).

A natureza pública da instituição, sua capacidade instalada e expertise de seus profissionais, e o volume de exames realizados (967 em 2012, média de 4 procedimentos/dia de operação) justificaram a escolha deste lócus. O tempo de instalação do equipamento e o volume de produção também permitem supor que a curva de aprendizado já foi ultrapassada e este estaria sendo utilizado na parte superior da curva de eficiência.

O sistema PET-TC foi adquirido por licitação em modalidade de compra turn key. Essa modalidade corresponde a um tipo de operação onde o vendedor fica obrigado a entregar o objeto de compra em condições de funcionamento, com o preço global do serviço e prazos para entrega 
definidos contratualmente no próprio processo 13. No caso do INCA, a aquisição envolveu um pacote de produtos e serviços, incluindo tomógrafo, sistemas computacionais de aquisição e processamento das imagens e outros acessórios; obras de adequação da infraestrutura, instalação e montagem; garantia integral de mão de obra, partes e peças; suporte técnico, atualização de softwares e hardwares por 30 meses, treinamento operacional in loco e na fábrica.

O INCA não possui fonte produtora do radiofármaco ${ }^{18} \mathrm{~F}-\mathrm{FDG}$ e depende de aquisição externa que, à época do estudo, derivava de um contrato com o Instituto de Engenharia Nuclear (IEN). Usualmente, são compradas de três a quatro doses/dia, com atividade radioativa de $60-70 \mathrm{mCi} /$ dose e valor fixo de $\mathrm{R} \$ 834,77 /$ dose, acrescidos dos custos de transporte. Podem ocorrer faltas ocasionais por problemas de produção levando a paralisações no funcionamento do serviço e a remarcações dos exames agendados.

\section{Definição do processo de produção do procedimento PET-TC}

Foi realizada através de visitas e entrevistas com a chefia do setor e profissionais envolvidos. Foi levantado o histórico de planejamento e instalação da tecnologia no INCA; as etapas componentes do processo de produção, e uma lista preliminar dos insumos físicos e humanos para sua realização, cujo consumo efetivo foi posteriormente quantificado nas observações diretas.

\section{Coleta de dados primários}

A coleta para as estimativas de custos foi realizada entre março e julho de 2012, correspondendo a 27 dias e cinco procedimentos em média por dia de observação. Foram acompanhados 85 procedimentos de aplicações oncológicas variadas, de pacientes elegíveis para a realização do exame, independente da localização da neoplasia, idade, sexo e peso. Critérios de exclusão compreenderam casos não-oncológicos, pacientes que faziam parte de protocolos de pesquisa ou que não realizaram o procedimento por causas diversas (ausência de jejum, glicemia sérica elevada e problemas técnicos com o 18F-FDG).

A duração de cada atividade do processo de produção, incluindo os tempos de deslocamentos dos pacientes e de trabalho de cada profissional envolvido, foi determinada por observação direta dos 85 exames.

Da mesma fora, para cada um desses procedimentos observados, foram verificados e quantificados todos os materiais consumidos em cada etapa.
Os dados coletados foram registrados em formulário específico, submetido a pré-teste, para ajustes e elaboração da versão final do instrumento. Este continha dois conjuntos de informações: (1) dados gerais por dia de observação (data da coleta; número de pacientes agendados e exames realizados; horário de chegada e atividade do radiofármaco na sua recepção; volume e número de doses recebidas; instituição fornecedora e valor pago pelo radioisótopo; duração do tempo de recepção do ${ }^{18} \mathrm{~F}-\mathrm{FDG}$ e dos testes diários do equipamento PET-TC); e (2) dados específicos de cada procedimento observado: (a) duração geral das etapas relacionadas ao procedimento em si e às atividades de apoio; (b) identificação dos profissionais e tempo de trabalho de cada categoria envolvida na produção do exame e atividades de apoio; (c) insumos fixos e variáveis consumidos. Aplicativo em programa Excel (Microsoft Corp., Estados Unidos) foi desenvolvido para registro dos tempos, insumos e valores correspondentes.

No período de coleta, não foi observada qualquer realização de procedimentos excepcionais (anestesia, uso de insulina em pacientes hiperglicêmicos ou de contraste para tomografia computadorizada).

\section{Quantificação e valoração dos recursos}

O tempo total do exame foi obtido a partir do horário de apresentação de cada paciente à recepção até sua liberação. A partir destas observações individuais, calculou-se o tempo médio e o desvio padrão de cada etapa. O tempo médio total do exame resultou do somatório dos tempos médios de cada etapa do procedimento.

Os tempos dedicados por cada categoria profissional, importantes na valoração dos gastos com recursos humanos, foram obtidos a partir do tempo da(s) etapa(s) em que o mesmo participava.

Os itens de custo identificados na realização do procedimento foram classificados em três categorias para análise: custos fixos, variáveis e semifixos 7. Custos fixos corresponderam à aquisição do equipamento de PET-TC e de outros insumos duráveis; custos de manutenção dos equipamentos; e custos operacionais, com estes últimos correspondendo a itens como luz, água, segurança, telefonia, limpeza do ambiente, descarte de resíduos. Custos variáveis envolveram a compra e transporte do radiofármaco e insumos médico-hospitalares. Custos com recursos humanos foram considerados como do tipo semifixo ${ }^{14}$, já que até certo número de procedimentos/dia não apresentavam variação e incidiam no custo, mesmo sem produção; a 
partir de certa faixa de volume de procedimentos, contudo, necessitarão ser elevados.

Os dados financeiros e contábeis para valoração dos insumos foram fornecidos pelos setores responsáveis pelo orçamento, planejamento e gestão do INCA e corresponderam aos últimos valores disponíveis em outubro de 2012.

O valor por dose do radiofármaco comprado do IEN foi R\$ 834,77, acrescidos de custos de transporte de R\$129,00, informações obtidas de notas fiscais e contratos de transporte disponíveis na Radiofarmácia e setor de Contratos e Convênios. Consumo dos demais insumos variáveis foi valorado de acordo com os dados da última compra, obtidos junto à Divisão de Suprimentos ou no portal de compras do Governo Federal (Sistema Comprasnet) 15.

Foram obtidas cópias dos documentos relativos à aquisição do equipamento em valores de dezembro de 2008. Estes valores foram atualizados para agosto de 2012, usando o Índice Geral de Preços (IGP-M) da Fundação Getúlio Vargas 16.

O pacote de aquisição, contudo, incluía um espectro variado de produtos e serviços. Alguns eram passíveis de depreciação, com grande variabilidade nos anos de vida útil, levando à tentativa de isolar os valores correspondentes aos diversos componentes do pacote.

O valor em reais da compra do equipamento, atualizado para agosto de 2012, foi subtraído do valor global do pacote e considerado, na sua depreciação, tempo de vida útil de sete anos.

Para estimativa dos valores do contrato de manutenção do equipamento foram obtidos os valores atuais mensais do contrato recém-negociado, cujo somatório para três anos, tempo de garantia de manutenção coberto no contrato original, foi deflacionado até dezembro de 2008 e subtraído do valor total restante de aquisição já excluído o valor do equipamento.

A quantia restante desta operação correspondia a itens que incluíam obras, mobiliário e equipamentos acessórios do PET-TC e da Radiofarmácia, bem como valores pagos para treinamento de pessoal, não sendo possível outras desagregações. Em função dessa variedade, que incluía elementos não depreciáveis, foi estabelecida depreciação do agregado considerando tempo de vida útil de 10 anos, menor que o usualmente estabelecido para obras e mobiliário.

Os valores de outros insumos fixos foram obtidos junto à Divisão de Suprimentos e no $s i$ te do Comprasnet ${ }^{15}$. Itens de mobiliário foram depreciados considerando vida útil de 10 anos, equipamentos de informática de 5 anos, fonte de calibração de 2,5 anos e artigos relacionados ao controle das radiações de 5 anos.
$\mathrm{Na}$ estimativa dos custos com recursos humanos, foram considerados o tempo dedicado de cada categoria profissional a cada uma das atividades do processo de produção do procedimento e de apoio.

Não é possível, contudo, estimar o quantitativo de horas de cada categoria profissional apenas se partindo dos somatórios dos tempos dedicados pelos indivíduos à produção do procedimento, em virtude das especificidades das legislações trabalhistas relacionados com o trabalho com radiação ionizante (que limita o regime de trabalho máximo em $24 \mathrm{hs}$ semanais e estabelece 20 dias consecutivos de férias por semestre) 17 . Respeitando estas, os dados obtidos foram utilizados para calcular uma equipe mínima dedicada necessária ao funcionamento do serviço de PET-TC, 5 dias na semana, 8 horas/dia, 240 dias/ ano. Esta equipe foi estimada como composta por 4 médicos nucleares, 2 enfermeiros, 2 radiofarmacêuticos, 2 físicos médicos, 2 técnicos de enfermagem, 2 técnicos em radiologia, 1 auxiliar administrativo e 1 recepcionista.

Como os profissionais do INCA são servidores da carreira de Ciência \& Tecnologia (C\&T) e recebem valores salariais diferenciados em relação aos demais vinculados ao Ministério da Saúde, foram construídas estimativas distintas dos custos do procedimento PET-TC, tomando por base os rendimentos salariais obtidos a partir da Tabela de Remuneração dos Servidores Públicos Federais, do Ministério do Planejamento, Orçamento e Gestão, na versão de julho de 201218. Foram consideradas as várias classes e padrões relacionados aos vencimentos básicos e às diferentes gratificações e, considerando a legislação trabalhista com radiação, aplicado adicional de radiação ionizante correspondente a $20 \%$ do vencimento básico 19 .

Para as estimativas dos custos operacionais (overhead), foram obtidas informações da área total da unidade HC I e as plantas do Setor de Medicina Nuclear, onde se situa o setor de PETTC. Os valores mensais de gastos com luz, água e esgoto foram rateados por $\mathrm{m} 2$ de área ocupada pelo setor de PET-TC. Custos de telefonia e segurança foram estimados baseando-se no número de telefones e de postos de trabalho existentes no mesmo. Custos de lavanderia e descarte de lixo consideraram quilos de roupa e litros de lixo não radioativo relacionados especificamente ao setor.

\section{Cenários de análise}

O caso de referência considerou pacientes oncológicos adultos, volume de produção de 5 exames/dia e uso de uma dose de 18F-FDG por pa- 
ciente. Construíram-se ainda dois cenários adicionais. Um deles, denominado funcionamento "pleno", considerava o aumento da produção para 10 exames/dia e uso de uma dose do radiofármaco por paciente. Tal estimativa tinha, como pressupostos, inexistência de dificuldades no fornecimento regular do radiofármaco e dedicação exclusiva dos diversos profissionais de saúde aos procedimentos PET-TC, o que não ocorre hoje. O outro, chamado "ideal", correspondeu à produção diária de 10 exames, uso de 0,6 dose/ paciente e recursos humanos remunerados exclusivamente pela carreira salarial do Ministério da Saúde.

Os custos totais anuais do procedimento foram estimados a partir do somatório dos seguintes itens de custos: (1) custo de capital do equipamento PET-TC; (2) adequação da área; (3) insumos duráveis e equipamentos auxiliares; (4) custo do 18F-FDG; (5) insumos médico-hospitalares; (6) recursos humanos, e (7) custos operacionais (overhead).

Foram também estimados os custos médios unitários, obedecendo à fórmula:

Cme $=\frac{C T_{p}}{y_{p}}$

onde, $\mathrm{Cme}=$ custo médio unitário; $\mathrm{CT}_{\mathrm{p}}=$ custos totais dada a produção de $\mathrm{p}$ procedimentos; $\mathrm{P}=$ número de procedimentos/dia (1 a 10); eY = produção anual dado p procedimentos/dia.

Para obter essas estimativas, o custo anual equivalente para os insumos duráveis (equipamento, mobiliário e infraestrutura física) foi aferido, segundo a seguinte fórmula:

$C A E=\frac{i^{*} X}{1-\frac{1}{(1+i)^{\mathrm{n}}}}$

onde, $\mathrm{CAE}=$ custo anual equivalente; $\mathrm{i}=$ tempo de vida útil do item; $\mathrm{X}=$ valor do item; e $\mathrm{n}=$ taxa de desconto (5\%).

\section{Análise de sensibilidade}

Foram empreendidas análises de sensibilidade univariadas que exploraram cenários mais e menos otimistas do consumo de recursos, considerando os seguintes parâmetros: (a) rendimento da dose de 18 F-FDG de $60 \%$, levando em conta o perfil de desempenho ótimo e menor tempo de aquisição de imagem do aparelho; (b) custo do radiofármaco variado entre $30 \%$ a menos e $15 \%$ a mais, mantidos valores de transporte do caso de referência; (c) salários considerando valores extremos das tabelas de rendimentos do Ministério da Saúde e C\&T; (d) tempo de depreciação do equipamento PET-TC entre 5 e 10 anos; (e) tempo de depreciação dos itens agregados do contrato de aquisição entre 5 e 20 anos; (f) variação dos insumos fixos e variáveis (excetuandose $18 \mathrm{~F}-\mathrm{FDG}$ ) para mais e menos $20 \%$; e, (g) taxa de desconto, utilizada no cálculo do custo anual equivalente, de 0 e $10 \%$.

Detalhamento dos parâmetros e variações utilizadas no caso de referência e nas análises de sensibilidade é apresentado na Tabela 1.

O estudo foi aprovado pelo Comitê de Ética e Pesquisa do INCA (registro no 165/11, CAE 0153.0.007.000-11).

\section{Resultados}

\section{Processo de produção do procedimento PET-TC}

Foram identificadas, a partir da observação direta, as seguintes etapas componentes do fluxo de produção:

- Recepção: compreendia a recepção, conferência dos dados e certificação do estado de jejum do paciente pela recepcionista, e seu encaminhamento ao local de espera da consulta.

- Anamnese pré-exame: realizada por profissional médico nuclear, incluía consulta clínica, verificação de tempo de jejum, orientações sobre o procedimento e seu planejamento, conforme indicação do exame.

- Preparo do paciente: realizada pela equipe de enfermagem, compreendia troca de roupa; aferição de peso e altura; mensuração da glicemia sérica; acomodação no leito nos "boxes" de repouso, obtenção do acesso venoso e instalação de hidratação venosa. A injeção do 18F-FDG delimitava o fim dessa atividade e início do tempo de repouso.

- Repouso: necessário à distribuição do 18F-FDG da corrente sanguínea para os órgãos, era monitorado pela equipe de enfermagem presencialmente e via câmeras de filmagem nos “boxes". Após 50 minutos, o paciente realizava esvaziamento vesical, completando-se os procedimentos de preparação do exame.

- Exame PET-TC: iniciava-se com o posicionamento do paciente na máquina pelo técnico em radiologia. Posteriormente, este realizava as atividades de programação da máquina, com aquisição e reconstrução das imagens.

- Avaliação da qualidade da imagem: realizada pelo médico nuclear, enquanto o paciente aguardava fora da sala de exame. Se a qualidade era satisfatória, era indicado o término do exame e o paciente tinha seu acesso venoso retirado e era liberado; caso contrário, retornava ao equipamento para nova sequência de aquisições. 
Parâmetros utilizados no caso de referência e nas análises de sensibilidade.

\begin{tabular}{|c|c|c|c|}
\hline \multirow[t]{2}{*}{ Variável } & \multirow[t]{2}{*}{ Caso de referência } & \multicolumn{2}{|c|}{ Variação } \\
\hline & & Pior cenário & Melhor cenário \\
\hline Número de procedimentos/dia & 5 & 3 & 10 \\
\hline Salários de carreira de Ciência \& Tecnologia (custo anual R\$) & $1.298 .044,35$ & $2.282 .855,23$ & $866.642,30$ \\
\hline Salários de carreira do Ministério da Saúde (custo anual R\$) & $1.030 .319,57$ & $1.276 .978,03$ & $820.647,60$ \\
\hline Rendimento do 18 F-FDG & 1 dose por procedimento & 1 dose por procedimento & 0,6 dose por procedimento \\
\hline Preço do 18F-FDG (R\$) & 834,77 & $+30 \%$ & $-15 \%$ \\
\hline Depreciação PET-TC (anos) & 7 & 5 & 10 \\
\hline Depreciação de acessórios do sistema + obras (anos) & 10 & 5 & 20 \\
\hline Custos fixos + operacionais & $1.428,50$ & $+20 \%$ & $-20 \%$ \\
\hline Insumos variáveis & 14,65 & $+20 \%$ & $-20 \%$ \\
\hline Taxa de desconto (\%) & 5 & 10 & 0 \\
\hline
\end{tabular}

18F-FDG: 18F-fluorodeoxiglicose; PET-TC: equipamento híbrido conjugando tomografia de emissão de pósitrons e tomografia computadorizada.

- Laudo do exame: elaborado por dois especialistas em medicina nuclear, compreendia a avaliação das imagens, demarcação das lesões identificadas, digitação dos achados e envio do laudo final.

- Atividades de apoio: incluíam: (1) controles do equipamento e do ambiente (calibração, checagem da estabilidade dos detectores individuais e verificação diárias dos sistemas automatizados do aparelho; checagem periódica do alinhamento adequado entre os scanners de imagem PET e TC etc.), a cargo do físico médico; e (2) à recepção e preparo do radiofármaco (recepção; dosagem da atividade radioativa, cálculo e fracionamento de dose, descarte dos resíduos), realizadas pelo radiofarmacêutico.

A Tabela 2 detalha a duração das atividades de realização do procedimento e de apoio, bem como os profissionais envolvidos e principais itens de custo de cada uma.

\section{Produção anual de procedimentos}

Linfomas Hodgkin e não-Hodgkin corresponderam a $34 \%$ das indicações dos exames observados, seguidos dos cânceres de pulmão (19\%) e colón e reto (12\%). Os tempos correspondentes às etapas observadas foram avaliados quanto à significância de eventuais desvios segundo a condição oncológica de base e os profissionais envolvidos, não sendo observadas diferenças de monta. Desse modo, nas estimativas foram utilizados os valores referentes à totalidade das observações.

O número total de procedimentos PET-TC considerando o caso de referência (5 procedi- mentos/dia) correspondeu a uma produção anual de 1.200 exames. A estimativa do rendimento máximo esperado do serviço, considerando condições atuais de infraestrutura física e pessoal disponível, permitiria o máximo de 10 exames/ dia e 2.400 procedimentos/ano.

\section{Custos do procedimento PET-TC}

As estimativas dos custos totais anuais de funcionamento do serviço de PET-TC e do custo médio por procedimento guardaram diferenças significativas de acordo com o regime salarial dos recursos humanos envolvidos (Tabela 3 ).

Para o caso de referência, o custo total anual do serviço de PET-TC, em valores de 2012 e considerando a realidade salarial da carreira de C\&T, foi de R\$ 3.780.356,88. Este custo era cerca de 270 mil reais inferior tomando por base os salários do Ministério da Saúde, em decorrência do significativo peso dos salários nos custos totais do procedimento, cinco pontos percentuais maior no primeiro caso. Isso se expressa também nos custos médios por procedimento: $\mathrm{R} \$$ $3.150,30$ e $\mathrm{R} \$ 2.927,19$, respectivamente.

Destaca-se a contribuição do radiofármaco nos custos totais do procedimento, que variou entre $26,5 \%$ e $28,5 \%$ dependendo do regime salarial. Nas estimativas realizadas com produção de 10 exames/dia, este item se torna o principal direcionador de custos em ambos os cenários salariais, contribuindo com mais de $40 \%$ do valor relacionado ao procedimento.

O volume de produção do serviço tem efeito expressivo no custo médio unitário do exame PET-TC. Para ambos os cenários salariais explo- 
Tabela 2

Processo de produção do procedimento PET-TC segundo duração média das etapas, profissionais envolvidos e principais itens de custo relacionados.

\begin{tabular}{|c|c|c|c|}
\hline Atividades & Duração média em minutos (DP) & Profissionais envolvidos & Principais itens de custo relacionados \\
\hline Recepção & $2(1,5)$ & Funcionário administrativo & $\mathrm{RH}$, estrutura física, mobiliário \\
\hline Anamnese & $6(1)$ & Médico nuclear & $\mathrm{RH}$, mobiliário \\
\hline Preparo & $69(8)$ & Equipe de enfermagem *, $* \star$ & $\mathrm{RH}, 18 \mathrm{FDG}$; insumos médico-hospitalares \\
\hline Repouso & $57(4)$ & Equipe de enfermagem $*, \star \star$ & $\mathrm{RH}$, estrutura física \\
\hline Exame PET-TC & $25(2)$ & Técnico de radiologia *** & Equipamento de PET-TC e acessórios \\
\hline Avaliação da qualidade & $15(2)$ & Médico nuclear & $\mathrm{RH}$, equipamentos acessórios \\
\hline da imagem & & & (computadores, softwares, estação de trabalho) \\
\hline Laudo & $45(20)$ & Médico nuclear & $\begin{array}{l}\text { RH, equipamentos acessórios (computadores, } \\
\text { softwares, estação de trabalho, rede de comunicação) }\end{array}$ \\
\hline Radiofarmácia & $28(9)$ & Radiofarmacêutico \# & $\begin{array}{l}\text { RH, estrutura física específica, } \\
\text { equipamentos de radiofármacia }\end{array}$ \\
\hline $\begin{array}{l}\text { Controles do equipamento } \\
\text { e do ambiente }\end{array}$ & $20(2)$ & Físico médico & $\begin{array}{l}\mathrm{RH} \text {, equipamentos de controle do equipamento e de } \\
\text { radiação ambiental (phanton, dosímetros etc.) }\end{array}$ \\
\hline
\end{tabular}

DP: desvio-padrão; 18F-FDG: 18F-fluorodeoxiglicose; PET-TC: equipamento híbrido conjugando tomografia de emissão de pósitrons e tomografia

computadorizada; RH: Recursos Humanos.

* Equipe de enfermagem inclui técnico de enfermagem e enfermeiro de nível superior;

** O médico nuclear, mesmo não participando diretamente do cuidado nestas etapas, necessita estar disponível para atendimento de intercorrências;

*** $O$ exame é realizado pelo técnico de radiologia sob supervisão do médico nuclear;

\# O físico nuclear é responsável pela supervisão do controle da radiação ambiental após etapas do dia envolvendo o $18 \mathrm{~F}-\mathrm{FDG}$.

rados, há uma redução progressiva do custo médio por exame entre um e 10 procedimentos/dia, até o limite da produção considerando as condições plenas de funcionamento na situação atual de recursos humanos e infraestrutura. A partir deste rendimento, há uma pequena elevação, decorrente principalmente da necessidade de ampliação da equipe de profissionais, com nova e progressiva queda, chegando a atingir, com 15 procedimentos/dia, os menores valores de custo unitário (respectivamente, $\mathrm{R} \$ 1.760,26$, no caso da carreira de C\&T, e R\$ 1.657,43, para valores salariais do Ministério da Saúde).

\section{Análises de sensibilidade}

Os parâmetros de incerteza do modelo foram submetidos à análise de sensibilidade univariada, de acordo com os intervalos explicitados na Tabela 1, determinando os valores de custo médio por procedimento dispostos na Tabela 4 . Verificou-se que, independente do perfil salarial considerado na análise, o elemento de maior impacto nos custos estimados do procedimento PET-TC correspondeu ao volume diário de produção dos exames.

Com vistas a determinar os fatores com maior impacto potencial nos custos estimados, foram elaborados Diagramas de Tornado pa- ra os dois cenários salariais (Figura 1). Para o perfil de salários C\&T, os parâmetros que mais influenciaram no custo unitário do exame foram a produção diária (49\%), as diferenças salariais entre os cargos e classes da carreira de C\&T (26\%) e a possibilidade de aproveitamento da dose do 18F-FDG (-11\%). Já para o perfil de salários do Ministério da Saúde, os parâmetros de produção diária (47\%), aproveitamento da dose $(-11 \%)$ e preço do 18F-FDG (-9\%) exerceram maior influência.

Em análise de sensibilidade bivariada contemplando a variação conjunta de (i) produção de 10 exames/dia; (ii) dose de Radiofármaco estimada em $60 \%$ do utilizado no caso de referência; e (iii) regime salarial do Ministério da Saúde, houve redução do custo médio por procedimento para $\mathrm{R} \$ 1.657,17$.

\section{Discussão}

A despeito de sua crescente aplicabilidade diagnóstica, um serviço de imagem PET permanece como extremamente complexo e custoso, reforçando a importância de estimativas de custo destes procedimentos.

Estudos de avaliação de custos dos procedimentos de PET são escassos. Duas pesquisas 
Tabela 3

Custos totais e médios do procedimento PET-TC para os cenários caso de referência e funcionamento pleno, por componente e tipo de custo, considerando as carreiras salariais de Ciência \& Tecnologia e de Saúde (em R \$ de 2012).

\begin{tabular}{|c|c|c|c|c|c|}
\hline \multirow[t]{2}{*}{ Itens de custo } & \multirow[t]{2}{*}{ Tipo de custo } & \multicolumn{2}{|c|}{ Caso de referência (5 exames/dia) } & \multicolumn{2}{|c|}{ Pleno funcionamento (10 exames/dia) } \\
\hline & & Custo anual (R\$) & Custo total (\%) & Custo anual (R\$) & Custo total (\%) \\
\hline \multicolumn{6}{|l|}{ Carreira de Ciência \& Tecnologia } \\
\hline Equipamento PET-TC * & Fixo & $838.259,17$ & 22,2 & $838.259,17$ & 17,5 \\
\hline Manutenção PET-TC ** & Fixo & $283.200,00$ & 7,5 & $283.200,00$ & 5,9 \\
\hline 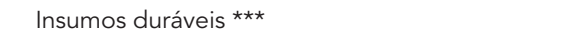 & Fixo & $261.186,15$ & 6,9 & $261.186,15$ & 5,4 \\
\hline Custos operacionais (overhead) \# & Fixo & $80.363,21$ & 2,1 & $80.363,21$ & 1,7 \\
\hline Insumos médico-hospitalares \#\# & Variável & $17.580,00$ & 0,5 & $35.160,00$ & 0,7 \\
\hline 18F-FDG \#\#\# & Variável & $1.001 .724,00$ & 26,5 & $2.003 .448,00$ & 41,7 \\
\hline Salários do Ministério de Ciência \& Tecnologia § & Semifixo & $1.298 .044,35$ & 34,3 & $1.298 .044,35$ & 27,0 \\
\hline Custo total anual & & $3.780 .356,88$ & 100,0 & $4.799 .660,88$ & 100,0 \\
\hline Custo médio unitário & & $3.150,30$ & & $1.999,86$ & \\
\hline \multicolumn{6}{|l|}{ Carreira do Ministério da Saúde } \\
\hline Equipamento PET-TC * & Fixo & $838.259,17$ & 23,9 & $838.259,17$ & 18,5 \\
\hline Manutenção PET-TC ** & Fixo & $283.200,00$ & 8,1 & $283.200,00$ & 6,2 \\
\hline Insumos duráveis *** & Fixo & $261.186,15$ & 7,4 & $261.186,15$ & 5,8 \\
\hline Custos operacionais (Overhead) \# & Fixo & $80.363,21$ & 2,3 & $80.363,21$ & 1,8 \\
\hline Insumos médico-hospitalares\#\# & Variável & $17.580,00$ & 0,5 & $35.160,00$ & 0,8 \\
\hline 18F-FDG \#\#\# & Variável & $1.001 .724,00$ & 28,5 & $2.003 .448,00$ & 44,2 \\
\hline Salários do Ministério da Saúde § & Semifixo & $1.030 .319,57$ & 29,3 & $1.030 .319,57$ & 22,7 \\
\hline Custo total anual & & $3.512 .632,10$ & 100,0 & $4.531 .936,10$ & 100,0 \\
\hline Custo médio unitário & & $2.927,19$ & & $1.888,31$ & \\
\hline
\end{tabular}

18F-FDG: 18F-fluorodeoxiglicose; PET-TC: equipamento híbrido conjugando tomografia de emissão de pósitrons e tomografia computadorizada.

* Equipamento, instalação, computadores, softwares com licença permanente, estação de rede;

** Com troca de peça original;

*** Acessórios do PET-TC, mobiliário, reforma, computadores, câmara plumbífera etc.;

\# Serviço de limpeza, coleta de resíduos, telefonia, segurança, lavanderia, luz, água e esgoto;

\#\# Luva, álcool 70, fita HGT, agulhas, gaze, algodão, jelco, solução fisiológica, equipo, seringa;

\#\#\# Radiofármaco e transporte;

$\S$ Médico, enfermeiro, farmacêutico, físico médico, técnico em radiologia e enfermagem, administrativo.

conduzidas na Bélgica por Krug et al. 4,5 adotaram o método de custeio por atividade (ABC). Outras abordagens, como a estimativa do custo para atender as exigências regulatórias para o funcionamento de serviços de PET, também têm sido realizadas em outros países 6 . Porém, estudos realizados no exterior não são transferíveis para nossa realidade, devido a diferenças nas estruturas de custos entre os sistemas de saúde, nos regimes salariais e nas legislações trabalhistas, que repercutem na operação dos serviços 20. Ainda que não seja factível comparação dos resultados desses estudos com os obtidos, é importante notar que todas estas estimativas também se basearam na identificação do processo de produção do exame e dos recursos necessários à sua realização.
Embora existam várias formas para estimar os custos unitários de um serviço em particular, a seleção do método mais apropriado depende do tipo de serviço, da razão para o custeio e da viabilidade econômica de se realizar seus cálculos 9. Nesse trabalho, foi utilizada a técnica de microcustos, partindo de uma abordagem baseada nos custos por atividade (ABC). Esta técnica é indicada em situações onde se desconhece os custos de uma nova tecnologia, onde a provisão dos serviços está baseada em um complexo arranjo organizacional (de modo que os inputs de custo podem variar entre as instituições) e onde os custos com recursos humanos e administrativos (overhead) representam parcela significativa dos custos totais 21 . Outra de suas vantagens é o alto grau de precisão com relação a um procedi- 
Custo médio por procedimento PET-TC segundo a variação nos parâmetros, na perspectiva de salários das carreiras de Ciência \& Tecnologia e do Ministério da Saúde.

\begin{tabular}{|c|c|c|c|c|}
\hline \multirow[t]{3}{*}{ Parâmetro } & \multicolumn{4}{|c|}{ Custo médio por procedimento PET-TC (em R\$) } \\
\hline & \multicolumn{2}{|c|}{ Carreira do Ministério da Saúde } & \multicolumn{2}{|c|}{ Carreira de Ciência \& Tecnologia } \\
\hline & Pior cenário & Melhor cenário & Pior cenário & Melhor cenário \\
\hline Número de procedimentos/dia & $4.312,37$ & $1.888,30$ & $4.684,21$ & $1.999,86$ \\
\hline Salários carreira de Ciência \& Tecnologia (custo anual) * & - & - & $3.970,97$ & $2.790,79$ \\
\hline Salários carreira Ministério da Saúde (custo anual) * & $3.132,74$ & $2.752,47$ & - & - \\
\hline Rendimento do 18 F-FDG *,** & - & $2.593,28$ & - & $2.816,39$ \\
\hline Preço do 18 F-FDG * & $3.052,41$ & $2.676,76$ & $3.275,51$ & $2.899,86$ \\
\hline Depreciação PET-TC * & $3.162,26$ & $2.752,11$ & $3.385,36$ & $2.975,21$ \\
\hline Depreciação de acessórios + obras * & $3.066,44$ & $2.859,59$ & $3.289,54$ & $3.082,69$ \\
\hline Custos fixos + operacionais * & $2.984,12$ & $2.870,27$ & $3.207,22$ & $3.093,37$ \\
\hline Insumos variáveis * & $2.930,12$ & $2.924,26$ & $3.153,23$ & $3.147,37$ \\
\hline Taxa de desconto * & $3.106,37$ & $2.763,87$ & $3.329,47$ & $2.986,97$ \\
\hline
\end{tabular}

18F-FDG: 18F-fluorodeoxiglicose; PET-TC: equipamento híbrido conjugando tomografia de emissão de pósitrons e tomografia computadorizada.

* Estimativas realizadas considerando produção de 5 exames/dia;

** Estimativa correspondente a realização de 5 procedimentos PET-TC com 3 doses de 18F-FDG (0,6 dose por procedimento).

mento específico, que permite que prescrições particulares sobre o mesmo possam ser delineadas. Por fim, além da análise da situação atual de uma atividade ou serviço, possibilitam ainda simulações nas variações dos recursos, gerando cenários diversos que expandem a compreensão e utilidade dos dados obtidos 5 .

Embora forneçam uma aproximação bem acurada dos custos reais, as estimativas de custos por essa metodologia são trabalhosas e frequentemente consomem bastante tempo e recursos, como se verificou neste estudo, e sua acurácia é contrastada pela necessidade de consistência e de generalização a outros contextos diferentes de onde foram realizadas. A despeito dessas desvantagens, a aplicação de microcustos é recomendada em situações onde o uso de recursos ou os custos unitários são elevados, porque os mesmos podem afetar significativamente os resultados de avaliações econômicas 20 .

A inexistência de informações sobre o custo do procedimento PET-TC no país dificulta a realização de análises de custo-efetividade da tecnologia. A pesquisa buscou minimizar essa lacuna, podendo servir de subsídio a estes estudos e nas decisões relativas à sua incorporação nas tabelas de reembolso do SUS. A metodologia e instrumentos desenvolvidos podem também ser apropriados por outros pesquisadores, auxiliando avaliações similares de custos do procedimento em tela e, nesse sentido, é possível a obtenção de informações detalhadas sobre o processo de produção dos resultados a partir de contato com os autores.

A opção pela perspectiva do SUS como provedor público de serviços determinou os custos levados em consideração 22 na pesquisa. Entretanto, as possibilidades de generalização (validade externa) dos resultados nessa perspectiva podem ser limitadas, em parte porque irão refletir as características dos arranjos institucionais locais 10. Esta limitação deve ser considerada quando da apreciação dos resultados e enseja a necessidade de outros estudos, realizados nos setores público e privado.

O INCA, embora órgão do SUS e unidade de referência da politica oncológica no país, apresenta peculiaridades, entre as quais seus servidores serem submetidos a um regime salarial específico e de maior remuneração. Na expectativa de aumentar a apropriação dos resultados e reduzir impactos das estimativas vinculadas a um provedor singular, foram contemplados dois cenários salariais. Isto resultou, para o caso de referência, em custos unitários do procedimento de $\mathrm{R} \$ 3.150,30$ na perspectiva salarial da C\&T e de $\mathrm{R}$ \$ 2.927,19 na do Ministério da Saúde.

O tempo de aquisição das imagens e a duração total do exame são aspectos críticos na produtividade dos serviços e seus custos. O equipamento disponível no INCA é um dos mais atualizados de sua categoria, tornando possível a realização dos exames com menor tempo de aquisição da imagem e menor atividade de 18 F-FDG. As esti- 
Figura 1

Diagramas de Tornado ilustrando análise de sensibilidade univariada dos diferentes efeitos dos parâmetros no custo unitário do procedimento PET-TC, considerando perspectiva de salários das carreiras.

1a) Carreira Ministério da Saúde

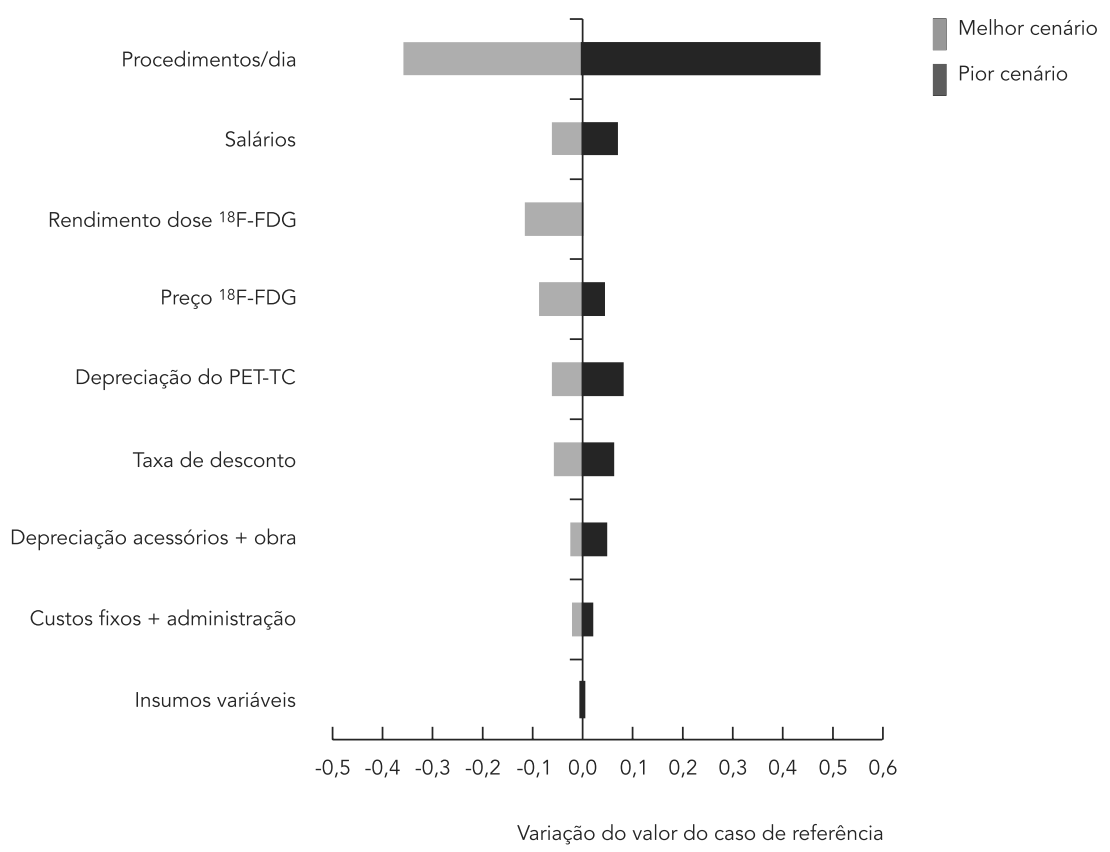

1b) Carreira Ciência \& Tecnologia

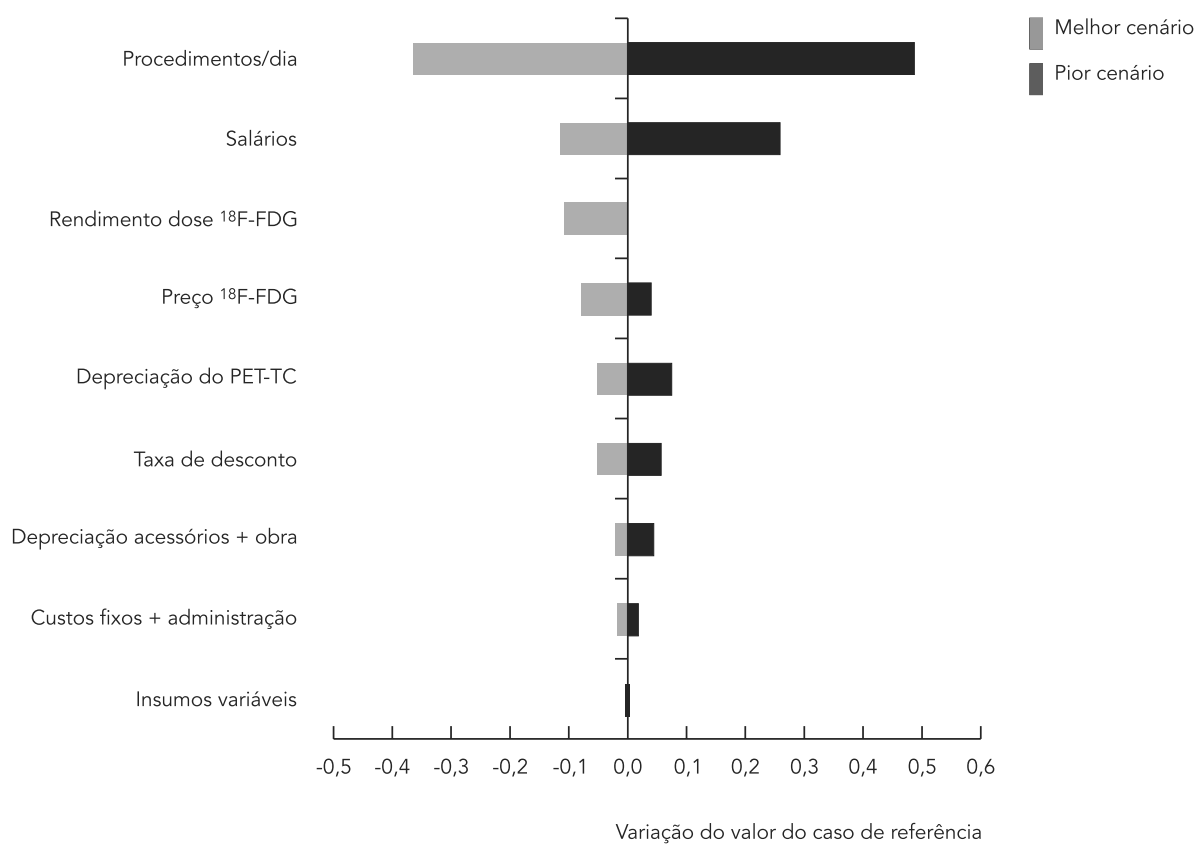

18F-FDG: 18F-flourodeoreiglicose; PET-TC: equipamento híbrido conjugando tomografia de emissão de pósitrons e tomografia computadorizada. 
mativas no caso de referência foram realizadas com uma dose por exame, com objetivo de gerar estimativas mais generalizáveis a outros locais com tomógrafos sem tal grau de desempenho. A situação de melhor aproveitamento da dose foi explorada na análise de sensibilidade, levando a redução significativa nos custos do procedimento: $\mathrm{R} \$ 2.816,39$ e $\mathrm{R} \$ 2.593,28$, de acordo com as duas realidades salariais (C\&T e Ministério da Saúde) consideradas.

Considerando o alto impacto dos valores de aquisição do ${ }^{18 \mathrm{~F}-F D G}$ nos custos totais, é razoável pensar que, à medida que sua produção comercial atinja maior escala, seu custo possa ser reduzido. O relatório de atividades do Comissão Nacional de Energia Nuclear (CNEN) de 201123 refere que o valor de venda do radiofármaco produzido pelo órgão encontrava-se, à época, $30 \%$ maior que o praticado no mercado privado nacional, em decorrência da multiplicação de produtores privados após fim do monopólio da União na produção de isótopos. A análise de sensibilidade contemplou esta possibilidade, considerando valor de compra $30 \%$ inferior, o que determinaria a queda nos custos médios do procedimento para $\mathrm{R} \$ 2.899,86$ (C\&T) e R $\$ 2.676,76$ (Ministério da Saúde).

Porque a maioria dos custos do procedimento são fixos ou semifixos, o volume de produção tem grande impacto nos custos anuais, que podem ser mais "diluídos" com maiores volumes de produção. A produtividade atual do serviço é de 5 exames/dia, mas ele foi originalmente projetado para realizar 20 procedimentos/dia, em dois turnos, situação inviável nas condições presentes de operacionalidade. Com vistas a explorar impacto de melhorias nessas condições, realizou-se uma exploração considerando 10 exames/dia, o que determinaria custos médios do procedimento de $\mathrm{R} \$ 1.999,86$ e $\mathrm{R} \$ 1.888,30$, considerando regimes salariais da C\&T e Ministério da Saúde. Situação semelhante foi observada por Chuck et al. 6 , em estudo no Canadá, que identificou variação nos custos por procedimento entre C\$ 7.869 (400 procedimentos/ano, 2 exames/dia) e C $\$ 1.231$ (3.220 exames/ano, 16 exames/dia).

O desempenho na capacidade inicialmente projetada exigiria a presença de um cíclotron in site para produção do radiofármaco ou seu fornecimento duas vezes/dia, por conta do decaimento da dose. Atualmente, esse fornecimento é definido contratualmente com o IEN, que possui apenas um cíclotron operando e produção limitada e extensiva a vários centros de imagem, limitando a quantidade fornecida a cinco doses/dia e constituindo-se em gargalo importante para atingir maior produtividade. Outro ponto de estrangulamento refere-se à escassez de recursos humanos. Esta produção exigiria uma equipe mínima exclusivamente dedicada ao procedimento maior que a atualmente disponível. Por fim, o volume de pacientes atendidos tem ainda como limitação o espaço físico, dado a necessidade de intervalo mínimo de 30-60 minutos entre a administração do radiofármaco e o início do exame para o equilíbrio orgânico do 18F-FDG. A disponibilidade de apenas três "boxes" onde o paciente deve guardar repouso delimita um tempo mínimo entre os procedimentos que não pode ser reduzido, mesmo com tempos de aquisição de imagem curtos. Assim, para que o serviço pudesse atender a demanda originalmente projetada, seriam necessárias mudanças nas características organizacionais, hoje limite concreto ao seu atingimento.

Ainda como parte das explorações sobre impacto das variáveis nos custos, foi simulado um cenário "ideal" que considerava aumento do rendimento para 10 exames/dia, aproveitamento de dose e funcionários sujeitos a regime salarial do Ministério da Saúde. Nesta situação, o custo médio do procedimento ficaria inferior a R\$ 1.700,00, representando decréscimo de $47 \%$ (carreira C\&T) e 43\% (Ministério da Saúde), respectivamente, em relação ao custo estimado do exame.

Em função da perspectiva adotada, os custos de capital configuram outra limitação à generalização dos resultados. A compra do equipamento pelo INCA foi feita pela modalidade de aquisição turn key, ainda pouco frequente no setor saúde brasileiro, mormente o público. Como os valores de aquisição levantados englobavam um elenco amplo de bens e serviços, que se estendiam para além do tomógrafo ou dos seus acessórios, buscou-se minimizar parte dessas restrições com o isolamento dos itens avaliados como mais relevantes - o equipamento per si e seu contrato de manutenção - e utilização de taxa de depreciação no restante do pacote menor que a usualmente aplicada a obras e instalações. Variação desse componente dos custos na análise de sensibilidade não resultou em mudanças significativas nos custos médios em nenhum dos dois cenários salariais examinados, como visto na Figura 1. Reforça-se, contudo, a importância de comparar as estimativas obtidas com outros estudos que examinem os itens de capital de forma mais desagregada.

Um elemento relevante em toda avaliação de custos refere-se à tentativa de validação dos resultados, mas a falta de estudos na realidade nacional dificulta estas comparações. A Classificação Brasileira Hierarquizada de Procedimentos Médicos (tabela CBHPM), elaborada pela Associação Médica Brasileira e Conselho Fede- 
ral de Medicina, estabelece valores de referência para remuneração de serviços profissionais prestados para o Sistema de Saúde Suplementar e leva em conta, dentre outros, depreciação do equipamento, aluguéis, manutenção, mobiliário e outras despesas associadas.

Segundo a última tabela vigente 24 , o valor do PET-TC seria de R\$3.116,06, a ser acrescido dos custos do radiofármaco e seu transporte. Trabalhando com os mesmos valores de dose e transporte utilizados na pesquisa (respectivamente, $\mathrm{R} \$ 834,77$ e $\mathrm{R} \$ 129,00$ ), o valor do procedimento ficaria em $\mathrm{R} \$ 4.079,83$ (variação entre $\mathrm{R} \$ 3.273,86-4.895,79$, considerando as bandas de $20 \%$ a mais e a menos que fazem parte da tabela). As estimativas apontam para um custo do procedimento no INCA que seria, respectivamente, $23 \%$ e $28 \%$ menor, com os regimes salariais de C\&T e do Ministério da Saúde. Mesmo considerando o limite inferior de $20 \%$ da tabela 24 , os valores estimados ainda seriam menores (3\% e $10 \%$, respectivamente). Na apreciação dessa comparação deve-se ter em mente que a tabela CBHPM tem por foco a remuneração do setor privado e, portanto, traz ainda embutido um percentual de lucro para o provedor do serviço, não contemplado nas estimativas aqui realizadas referente a um provedor público.

\section{Resumen}

La tomografía por emisión de positrones (PET) es de uso reciente en Brasil y es necesario estimar sus costes, con el fin de subsidiar estudios de evaluación económica sobre esta tecnología. El trabajo examina el proceso de producción de PET-TC con 18F-FDG y se estimaron sus costes desde la perspectiva de un prestador público de servicios de salud. Se empleó la técnica de microcostes, con la identificación, cuantificación y valoración de los insumos consumidos en la producción del procedimiento. Las estimaciones consideran la observación de 85 exámenes entre marzo y julio de 2012. El "caso base" considera pacientes adultos de cáncer, con una producción de 5 exámenes/día y el uso de una dosis
Além disso, o setor privado tem liberdade de compra do radiofármaco maior que o INCA, podendo facilitar negociações em relação ao preço desse insumo. Refazendo as estimativas com uma redução de $30 \%$ no valor da dose de 18 F-FDG, os valores de referência do procedimento para a tabela CBHPM ficariam em torno de R $\$ 3.829,39$. Ainda assim, os valores estimados para o INCA seriam inferiores: 18\% (salários C\&T) e $24 \%$ (Ministério da Saúde).

\section{Conclusões}

Uma análise financeira é tão mais acurada quanto seus pressupostos de base e nenhum modelo descreve a realidade perfeitamente. Diversos itens relacionados à tecnologia de imagem vêm se alterando nos últimos tempos, como o preço dos equipamentos e do radiofármaco, que têm decaído à medida que a tecnologia entra em fase de maior estabilização de design e que aumenta a produção comercial privada do radioisótopo.

Este trabalho permitiu estimar um valor de referência a ser utilizado em avaliações de custoefetividade e a exploração de um conjunto de elementos que podem impactar no custo do exame nas instituições públicas de saúde. Outros estudos, contudo, se fazem necessários com vistas a ultrapassar algumas das limitações apontadas. de $18 F-F D G$ por paciente. Los costes unitarios del procedimiento fueron, respectivamente, $R \$ 3.150 .30$ y $R \$$ 2.927.19, desde la perspectiva del Ministerio de Ciencia y Tecnología y del Ministerio de Salud. El volumen diario de producción fue el elemento de mayor impacto en los costes. Además, se analizaron los factores que pueden repercutir en el coste del examen en instituciones de salud pública.

Tomografia de Emisión de Positrones; Tecnología Biomédica; Costos y Análisis de Costo; Tecnología de Alto Costo 


\section{Colaboradores}

R. Caetano colaborou na concepção do estudo, análise e interpretação dos dados, redação do artigo e aprovação da versão final. L. F. Schluckebier participou do desenho das ferramentas de pesquisa, coleta e interpretação dos dados, redação do artigo e aprovação da versão final. C. R. G. Bastos esteve envolvida na coleta dos dados, redação do artigo e aprovação da versão final. R. M. Silva contribuiu no pré-teste dos instrumentos de coleta, na redação do artigo e aprovação da versão final. M. P. Carneiro participou na análise dos resultados, redação do artigo e aprovação da versão final. J. W. E. Silva contribuiu na elaboração e revisão dos instrumentos de coleta, discussão dos resultados obtidos e redação da versão final. A. N. Biz trabalhou nas etapas de desenho das ferramentas de pesquisa, coleta e interpretação dos dados, redação do artigo e aprovação da versão final.

\section{Referências}

1. Vianna CMM, Caetano R. Avaliações econômicas como um instrumento no processo de incorporação tecnológica em saúde. Cad Saúde Colet (Rio J.) 2005; 13:747-66.

2. Ministério da Saúde. Política de gestão de tecnologias em saúde. Brasília: Ministério da Saúde; 2011.

3. Brasil. Lei no 12.401, de 28 de abril de 2011. Altera a Lei no 8.080, de 19 de setembro de 1990, para dispor sobre a assistência terapêutica e a incorporação de tecnologia em saúde no âmbito do Sistema Único de Saúde. Diário Oficial da União 2011; 29 abr.

4. Krug B, Zanten AV, Pirson AS, Crott C, Borght TV. Activity-based costing evaluation of [18F]-fludeoxyglucose production. Eur J Nucl Med Mol Imaging 2008; 35:80-8.

5. Krug B, Zanten AV, Pirson AS, Crott C, Borght TV. Activity-based costing evaluation of a $[18 \mathrm{~F}]$-fludeoxyglucose positron emission tomography study. Health Policy 2009; 92:234-43.

6. Chuck A, Jacobs P, Logus JW, St. Hilaire D, Chmielowiec C, McEwan AJ. Marginal cost of operating a positron emission tomography center in a regulatory environment. Int J Technol Assess Health Care 2005; 21:442-51.

\section{Agradecimentos}

À pesquisadora Marcia Teixeira Pinto, do Núcleo de Avaliação de Tecnologias em Saúde, Instituto Nacional de Saúde da Mulher, da Criança e do Adolescente Fernandes Figueira, que colaborou com sua expertise nas fases iniciais de desenho do estudo e desenvolvimento dos formulários de pesquisa, e ao Setor de Medicina Nuclear do Instituto Nacional de Câncer, que gentilmente disponibilizou as instalações e foram extremamente colaborativos durante as etapas de coleta de dados e discussão dos resultados da pesquisa.
7. Drummond M, Sculpher MJ, Torrance GW, O'Brien B, Stoddart GL. Methods for the economic evaluation of health care programmes. 3rd Ed. Oxford: Oxford University Press; 2005.

8. Ridderstolpe L, Johansson A, Skau T, Rutberg H, Ahlfeldt $\mathrm{H}$. Clinical process analysis and activitybased costing at a heart center. J Med Syst 2002; 26:309-22.

9. Krug B, Pirson AS, Crott R, Borght TV. Is a methodology available that accurately measures the cost of an FDG-PET study? Eur J Nucl Med Mol Imaging 2007; 34:625-7.

10. Muennig P. Cost-effectiveness analysis in health A practical approach. 2nd Ed. New Jersey: JosseyBass; 2008.

11. Gold M. Panel on cost-effectiveness in health and medicine. Med Care 1996; 34(12 Suppl):DS197-9.

12. Player S. Activity-based analyses lead to better decision making. Healthc Financ Manage 1998; 52:66-70. 
13. Fontoura PS. Estudo de caso de utilização de "Extranet” na gestão do processo de administração de contratos EPC (Engineering, Procurement and Construction) na modalidade "Turn key" [Dissertação de Mestrado]. Curitiba: Universidade Federal do Paraná; 2006.

14. Ministério da Saúde. Programa Nacional de Gestão de Custos: manual técnico de custos - conceitos e metodologia. Brasília: Ministério da Saúde; 2006.

15. Ministério do Planejamento, Orçamento e Gestão. Portal de compras do Governo Federal - Comprasnet. http://www.comprasnet.gov.br (acessado em 13/Set/2012).

16. Banco Central do Brasil. Calculadora do cidadão. Aplicativo de correção de valores. https://www3. bcb.gov.br/CALCIDADAO/publico/exibirForm CorrecaoValores.do?method=exibirFormCorrecao Valores\&aba $=1$ (acessado em 13/Set/2012).

17. Brasil. Decreto oㅡ 84.106, de 22 de outubro de 1979. Altera a redação do art. 7o do Decreto no 81.384 , de 22 de fevereiro de 1978, que dispõe sobre a concessão das vantagens previstas na Lei no 1.234, de 14 de novembro de 1950. Diário Oficial da União 1979; 23 out.

18. Ministério do Planejamento, Orçamento e Gestão. Tabela de remuneração dos servidores públicos federais, v. 59. http://www.servidor.gov.br/publi cacao/tabela_remuneracao/tab_remuneracao/ tab_rem_12/tab_59_2012a.pdf (acessado em 10/ Jan/2013).
19. Brasil. Decreto oo 877, de 20 de julho de 1993. Regulamenta a concessão do adicional de irradiação ionizante de que trata o parágrafo lo do art. 12 da Lei no 8.270, de 17 de dezembro de 1991. Diário Oficial da União 1993; 21 jul.

20. Sculpher MJ, Pang FS, Manca A, Drummond MF, Golder S, Urdahl H, et al. Generalisability in economic evaluation studies in healthcare: a review and case studies. Health Technol Assess 2004; 8:iiiiv, 1-192.

21. Mogyrosy Z, Smith P. The main methodological issues in costing health services: a literature review. York: Centre for Health Economics, University of York; 2005. (CHE Research Paper, 7).

22. Ministério da Saúde. Diretrizes metodológicas: estudos de avaliação econômica de tecnologias em saúde. Brasília: Ministério da Saúde; 2009.

23. Comissão Nacional de Energia Nuclear. Relatório de gestão do exercício de 2011. Rio de Janeiro: Comissão Nacional de Energia Nuclear; 2012.

24. Associação Médica Brasileira. Classificação brasileira hierarquizada de procedimentos médicos. São Paulo: Câmara Brasileira do Livro; 2012.

Recebido em 30/Jan/2013

Versão final reapresentada em 24/Jul/2013

Aprovado em 26/Ago/2013 